\title{
Research Progress on Continuous Cropping Obstacle and Green Control of Strawberry
}

\author{
Zhengwei Xie ${ }^{\mathrm{a}}$, Qianqian Ma ${ }^{\mathrm{b} *}$, Wanyun Peng ${ }^{\mathrm{c}}$, Zhide Wang ${ }^{\mathrm{d}}$, Peng Wu $\mathrm{W}^{\mathrm{e}}$, Yexing Sun ${ }^{\mathrm{f}}$ \\ Dazhou Academy of Agricultural Sciences, Dazhou, Sichuan, China
}

\begin{abstract}
Continuous cropping obstacle is a big problem of Strawberry planting. Continuous cropping obstacle leads to the accumulation of phenolic acids, imbalance of soil microorganism, deterioration of physical and chemical properties, resulting in sharp decline in Strawberry yield and quality. At present, the prevention and cure of continuous cropping obstacle of Strawberry is an urgent problem to be solved. The pathogen does not produce drug resistance, is safe to fresh fruit and does not pollute the environment.
\end{abstract}

\section{Introduction}

Strawberry is one of the most widely cultivated fruit trees in the world. It is known as "the Queen of Berries". In 2018, the world strawberry planting area is 372,400 $\mathrm{hm}^{2}$, the annual yield is 8337,100 tons. In 2017, the world imported 947,500 tons of strawberries and exported 951,400 tons. China is the largest strawberry producing country in the world, and its annual output has been the first in the world since 1994. In 2018, china planted $111,100 \mathrm{hm}^{2}$ in strawbery, and its annual output is 2964,300 tons, accounting for $1 / 3$ of the whole world. The planting area gradually expanded, but there was a contradiction between the rotation of land and fixed facilities, and the continuous cropping obstacle became increasingly obvious.

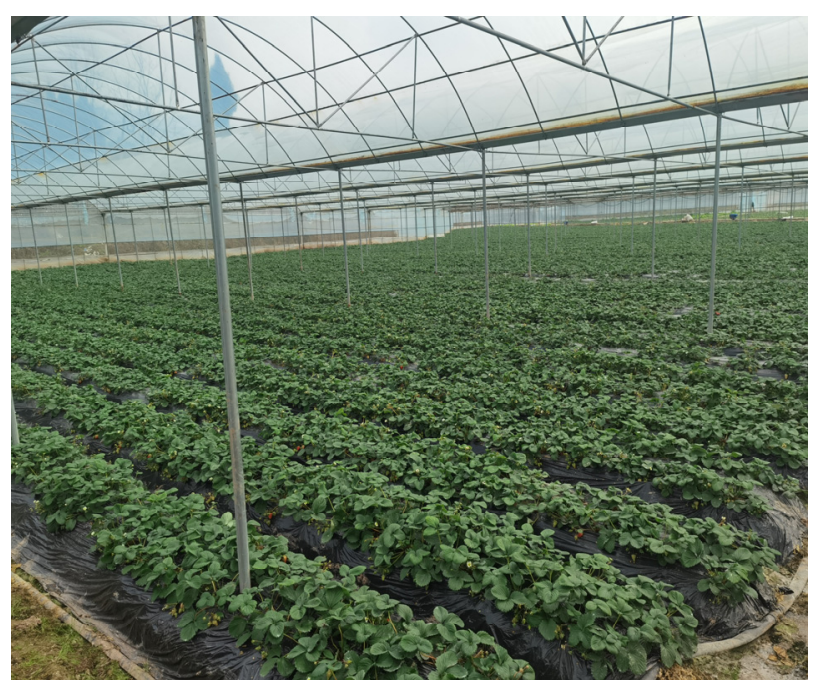

The continuous cropping disease of strawberry was the main factor restricting strawberry production at present, and the disease was prevalent in strawberry producing areas all over the world, the occurrence of the disease, the light leading to yield reduction and yield reduction, heavy or no harvest, serious constraints on the sustainable development of strawberry. In the past, chemical bactericides were widely used to disinfect the soil, but the chemical disinfecting of the soil would pollute the environment in different degree. In this paper, the continuous cropping obstacle of strawberry and the measures of green control were summarized in order to provide reference for further research and solution of continuous cropping obstacle of strawberry.

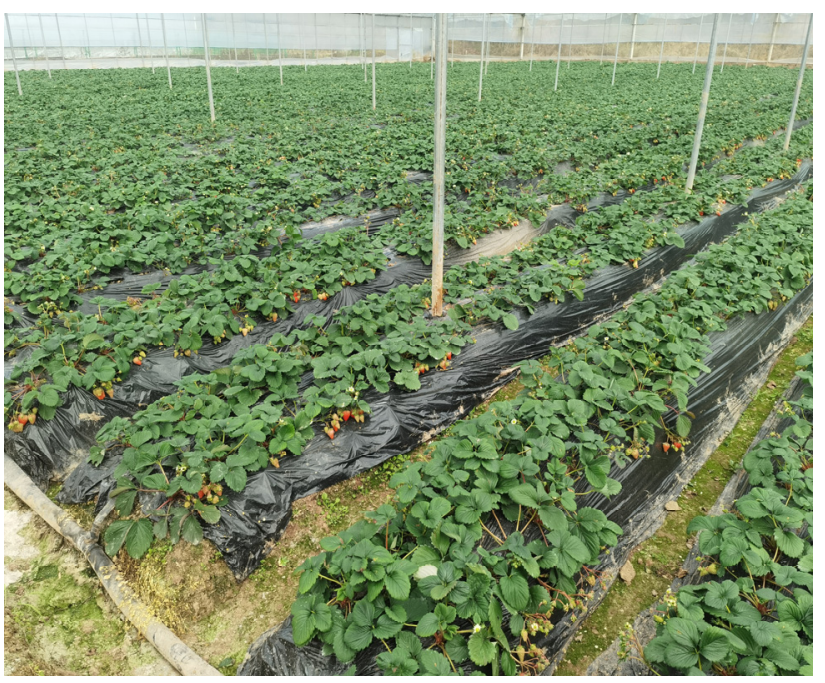

\footnotetext{
* QianqianMa: 2282583901.qq.com

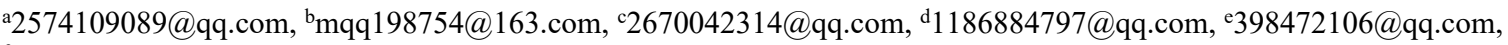

f745358474@qq.com
} 


\section{The cause of continuous cropping obstacle of strawberry}

\subsection{Plant autotoxicity}

The autotoxicity of plants is that some substances can be released from some plants by means of above-ground leaching, root exudates and plant stubble, etc., autotoxicity is a kind of growth inhibition that occurs within species. Soil ecological environment has a great influence on plant growth under continuous cropping conditions, especially the metabolites of plant residues and pathogenic microorganisms are toxic to plants, and together with the autotoxic substances secreted by the plant root affect plant metabolism, resulting in the occurrence of autotoxicity.

Strawberry root exudates contain phenolic acids, such as p-hydroxybenzoic acid and cinnamic acid, which strongly inhibit the growth of plants. The results showed that the contents of p-hydroxybenzoic acid, cinnamic acid, ferulic acid and coumaric acid in soil increased significantly with the increase of continuous cropping time of strawberry, especially p-hydroxybenzoic acid [13]. The results showed that phenolic acid allelochemicals could inhibit the growth of strawberry roots, decrease the content of chlorophyll and the activity of SOD in leaves, and decrease the disease resistance of strawberry [4]. This indicated that the autotoxicity of allelochemicals was an important cause of continuous cropping disorder of strawberry.

\subsection{Deterioration of soil biological environment}

The results showed that the number of nitrite bacteria and nitrate bacteria in greenhouse soil was higher than that in open soil, and the content of accumulated nitrate nitrogen was higher and increased gradually with the increase of temperature. The species and quantity of soil harmful fungi increased obviously with the increase of planting years in protected field, and the disease accounted for about $85 \%$ of all continuous cropping obstacles [5], especially soil disease was the main factor of continuous cropping obstacles, and some harmful fungi that have never been found can also have an adverse effect on crop roots.

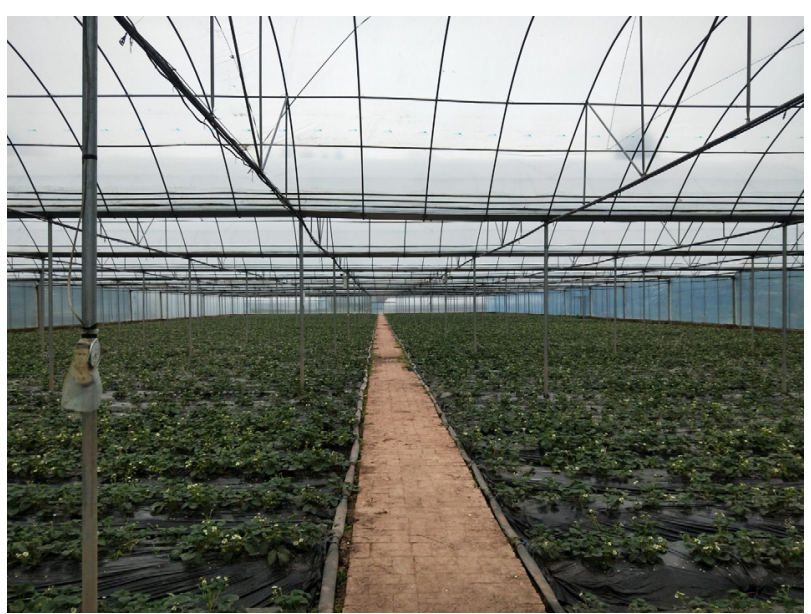

\subsection{Soil acidification and salinization}

Long-term large-scale use of chemical fertilizer, of which only part is absorbed by crops, and most of the residual soil, which is the main reason for the decline of soil $\mathrm{pH}$ and secondary salinization, and because of the high temperature and humidity in the shed, the rain water has little leaching effect, with the increase of cultivation years, the accumulation of acid root in the topsoil was serious, and the strawberry took a lot of calcium, magnesium and potassium from the soil, which caused the soil nutrient imbalance and made the basic elements such as calcium and magnesium in the soil colloid easy to be converted, soil acidification, soil moisture evaporation, soil moisture in the lower soil and soil moisture evaporation from the deep to the soil surface migration of salt, resulting in the soil surface salt accumulation, inhibition of the absorption of boron, calcium, manganese, zinc and other nutrients causes the imbalance between soil nutrients, easy to be absorbed by strawberry nutrients decreased year by year, resulting in imbalance of nutrient balance around strawberry rhizosphere, causes the strawberry nutrient deficiency to cause the abnormal fruit, reduces the production.

\subsection{The balance of soil elements is disturbed}

Facility strawberries grow in the same place for many years, disrupting the balance of nutrients in the soil. Strawberry is a shallow-root crop, which absorbs more nutrient elements in the surface of soil and selectively absorbs nutrient elements. Planting strawberry in the same place continuously for a long time inevitably results in the deficiency of one kind of nutrient elements, the accumulation of the other nutrient elements resulted in the decrease of the nutrient elements near the root and the accumulation of the non-selected elements, which affected the root growth. 


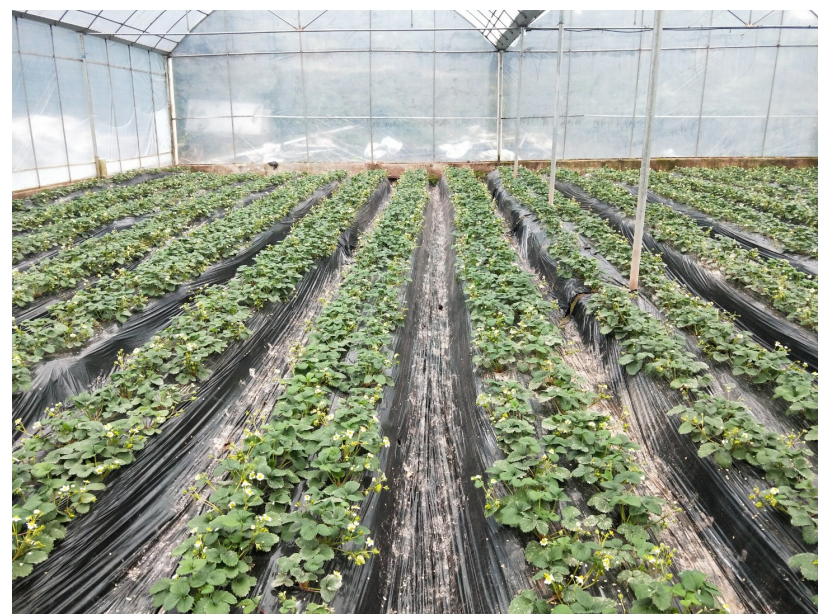

\section{Green control of continuous cropping obstacle of Strawberry}

\subsection{Promotion and application of non-toxic seedlings}

Strawberry is a perennial plant fruit tree, farmers generally choose self-breeding seedlings, with the increase in the number of breeding years, carrying pathogens increased year by year, for carrying virus disease, there is no chemical can effectively control. Therefore, the cultivation of virus-free parent seedlings and virus-free seedlings is the fundamental strategy to control strawberry virus disease. The virus, fungi and bacteria of most plants can be removed by tissue culture, so that the plants grow strongly, the flowers grow bigger, the color is bright, the ability of resistance to disease and adversity is improved, and the number of flowers and fruits is increased. In addition, tissue culture can shorten the propagation period of strawberry and improve the propagation ability and coefficient of strawberry. At the same time, tissue culture belongs to vegetative propagation, which keeps the good character of the female parent.

\subsection{Application of organic amendments}

After strawberry harvest, the soil was treated with chitin powder, plant residues, vermicompost, green manure, cake fertilizer, rice straw, compost and manure, which could improve the soil structure and nutrient conditions of soil microorganisms, improve soil microbial diversity, degradation of volatile substances, thus inhibiting the growth of pathogenic bacteria. The control effect of organic amendments on different diseases was different, the order was scab, pythium, phytophthora, fusarium wilt and rhizoctonia solani.

\subsection{Solar thermal sterilization}

Solar energy is a cheap, safe, simple and practical method of soil disinfection, which has been widely used in facility cultivation in recent years. First of all, clean up the weeds in the field, tillage after the border, select high temperature weather, in the border furrow irrigation. The amount of irrigation water is suitable for the soil to be in the state of saturated water, making the soil in the state of anoxic, which is favorable for the elimination of pathogenic bacteria in the soil [6]. After irrigation, the greenhouse was closed and the soil temperature was above $40^{\circ} \mathrm{C}$ for $240 \sim 360 \mathrm{~h}$, and the cover was removed.

\subsection{Soil steam sterilization}

Most soil-borne pathogenic fungi and weeds can be effectively controlled by soil treatment with steam at $80 \sim 100^{\circ} \mathrm{C}$. Worldwide, steam is increasingly being used as an alternative to soil sterilization. Some new and more efficient ways of using steam are being studied [7]. In some cases, the release of toxic compounds may inhibit plant growth or kill beneficial fungi, such as mycorrhizal fungi. Irrigation before planting can reduce the toxicity caused by steam fumigation. However, due to the high cost of soil steam sterilization, generally only in greenhouse conditions, the production of high economic value crops only used.

\subsection{Biocontrol agents}

Biocontrol bacteria could antagonize pathogens and degrade root exudates such as phenolic acids in continuous cropping strawberry, which enhanced the quality of strawberry significantly [8-9]. Biocontrol agents have obvious advantages in alleviating diseases and insect pests caused by continuous cropping, bacillus, pseudomonas, paenibacillus and actinomycetes are commonly used in biological control of continuous cropping diseases and insect pests. Inoculating actinomycetes can effectively change the microbial ecological environment of strawberry root zone, and then alleviate the continuous cropping obstacle of strawberry bacillus subtilis can inhibit the growth of strawberry root rot Fusarium oxysporum and Botrytis cinerea [10-11].

\subsection{Utilization of plant derived extracts}

It also has great potential to use plant extracts to control continuous cropping obstacle of strawberry. At present, 16 kinds of plant extracts have been screened out, which can reduce the rate of dead seedlings by $22.4 \% \sim 33.2 \%$, increase output $32.6 \% \sim 33.8 \%$. The inhibitory effect on mycelium growth of fusarium graminearum was more than $50 \%$, and the inhibitory activity of glycyrrhiza uralensis extracts was $86.99 \%, 23$ kinds of chinese herbal extracts had more than $50 \%$ inhibitory effect on the mycelium growth of rhizoctonia solani, and the inhibitory rate of celosia cristata extract was 100\% [12]. The water extracts of garlic and leek and the water decoctions of cloves and rhubarb had strong inhibitory effect on colletotrichum gloeosporioides in higher concentrations, and the water decoctions of forsythia suspensa had strong inhibitory effect on colletotrichum gloeosporioides in different concentrations. Lavender as the basic material, can effectively reduce the number of 
ergot grains of strawberry wilt pathogen verticillium dahliae [13].

\subsection{Selection of new disease-resistant varieties}

Now the plant hybridization technology has also been mature, can selectively breeding disease and pest resistance of new strawberry varieties to promote cultivation. The results showed that used mutation breeding technology, combined with plant tissue culture, used fusarium oxysporum F. Sp. fusarium crude toxin as screening agent to screen fusarium oxysporum mutants [14-15].

\section{References}

1.Asao T, Kitazawa H, Ban T, et al. Electrodegradation of root exudates to mitigate autotoxicity in hydroponically grown strawberry plants [J]. Hortscience, 2008, 43(07): 2034-2038.

2.Kitazawa H, Asao T, Ban T, et al. Autotoxicity of root exudates from strawberry in hydroponic culture $[\mathrm{J}]$. The Journal of Horticultural Science and Biotechnology, 2005(80): 677-680.

3.W.C. Zhen, X.Y. Wang, J.Y. Kong, et al. Determination of phenolic acids in root exudates and decomposing products of strawberry and their allelopathy [J]. Journal of Agricultural University of Hebei, 2004, 27 (04): 74-78.

4.Z.H. Gao, X.Y. Zhang, H.B. Ge, et al. Modeling the obstacle effects of strawberry root exudates [J]. Plant Nutrition and Fertilizer Science, 2008, 14(01): 189-193.

5.Koitabashi R, Suzuki T, Kawazu T, et al. 82Cineole inhibits root growt hand DNA synthesis in the root apical meristem of Brassica campest ris L1 [J]. J. Plant Res., 1997, 110(1097): 1-6.

6.P. Wang. Occurrence mechanism and control strategy of continuous cropping obstacle of strawberry in protected field [J]. Chinese Gardening Digest, 2012, 28(7): 178-179.

7.W.C. Zhen, K.Q. Cao, T.L. Hu. The rese arch advance on cropreplant diseases [J]. Journal of Agricultural University Of Hebei, 2004, 20(4): 98-102.

8.Y.J. Xu, Q.H. Xue, S.L. Xing, et al. The growth promoting effect and induced endurance of three actinomyces strains to strawberry [J]. Acta Agriculturae Boreali-Occidentalis Sinica, 2007, 16(06): 146-153.

9.Q. Xue, H.X. Lai. Effects of actinomycetes preparation on the flower and fruitage of strawberry [J]. Northern Horticulture, 2010(06): 177-179.

10.X.J. Wang, C.Y. Bu, Y.S. Jin, et al. Identification and inhibitory effects of antagonistic bacteria against strawberry root rot (StrawberryDuch.) [J]. Acta Horticulturae Sinica, 2011, 38(09): 1657-1666.

11.D.Q. Li, Y.M. Qian, M.M. Zhou, et al. The mechanism of biological control of strawberry gray mould using the marine bacterial NH-8 strain and analysis of the antifungal substances from the strain [J]. Acta Phytophylacica Sinica, 2016, 43(02): 215 221.

12.S.T. Wang, F.Q. Zhang, R.P. Gao, et al. Inhibition of 126 Kinds of Chinese Herb Extracts Gainst Two Plant Pathogenic Fungi [J]. Journal of Henan Agricultural Sciences, 2006(10): 62-65.

13.Yohalem D, Passey T. Amendment of soils with fresh and post - extraction lavender (Lavandula angustifolia) and lavandin (Lavandula intermedia) reduce inoculum of Verticillium dahliae and inhibit wilt in strawberry [J]. Applied Soil Ecology, 2011, 49: 187-196.

14.Y.H. Ma. Screening of resistant mutants to continuous cropping obstacles in strawberry and resistance evaluations of their regeneration plants [D]. Hebei of China: Agricultural University of Hebei Province, 2012.

15.X.J. Liu. In vitro selection of mutants to wilt disease resistant and studies on their resistance mechanisms in strawberry [D]. Hebei of China: Agricultural University of Hebei Province, 2009. 